\title{
Generalization of Common Fixed Point Theorems for Two Mappings
}

\author{
Mumtaz Ali ${ }^{*}$, Muhammad Arshad \\ Department of Mathematics, International Islamic University, Islamabad, Pakistan \\ *Corresponding author: mumtaz6767@yahoo.com
}

Received August 07 2017; Revised December 01, 2017; Accepted December 23, 2017

\begin{abstract}
In this paper we study and generalize some common fixed point theorems in compact and Hausdorff spaces for a pair of commuting mappings with new contraction conditions. The results presented in this paper include the generalization of some fixed point theorems of Fisher, Jungck, Mukherjee, Pachpatte and Sahu and Sharma.
\end{abstract}

Keywords: contraction mapping, fixed point, compact metric space

Cite This Article: Mumtaz Ali, and Muhammad Arshad, "Generalization of Common Fixed Point Theorems for Two Mappings.” Turkish Journal of Analysis and Number Theory, vol. 5, no. 6 (2017): $230-239$. doi: 10.12691/tjant-5-6-5.

\section{Introduction}

Fixed point theorey is a fascinating topic for research in modern analysis and topology. The study and research in fixed point theory began with the pioneering work of Banach [2], who in 1922 presented his remarkable contraction mapping theorem popularly known as Banach contraction mapping principle. It has widespread applications in both pure and applied mathematics. In 1961, Edelstein [8] for the first time introduced the concept of contractive mapping defined on compact metric spaces. According to Edelstein "if $T$ is a continuous mapping of a compact metric space $X$ into itself satisfying $d(T x, T y)<d(x, y)$ for all $x, y \in X$, $x \neq y$, then $T$ has unique fixed point in $X$ ". In 1976, Jungck [16] generalized contraction mapping theorem for a pair of commuting mappings. Later on several mathematicians have been generalized and improved The Banach contraction mapping theorem for fixed points in several different ways viz, Bondar [3], Browder [4], Chatterjee [5], Ciric [6] are a few to name. For more results in this direction, we refer to [12,13,19,20,22,23] and references therein. In this paper we want to establish fixed point results in complete, compact and Hausdorff spaces for a pair of commuting mappings. The obtained results are generalizations of some fixed point theorems of Fisher [9], Jungck [16], Mukherjee [17], Pachpatte [18] and Sahu and Sharma [21].

The following fixed point theorems were proved in $[9,16,17,18]$ and [21].

Theorem 1.1. [9] Let $T$ be a mapping of the complete metric space $X$ into itself satisfying the inequality

$$
\begin{aligned}
& (d(T x, T y))^{2} \\
& \leq \alpha_{1}(d(x, T x) d(y, T y))+\alpha_{2}(d(x, T y) d(y, T x))
\end{aligned}
$$

$\forall x, y \in X, \quad 0 \leq \alpha_{1}<1$ and $0 \leq \alpha_{2}$ then $T$ has a fixed point in $X$.

Theorem 1.2. [16] Let $f$ be a continuous mapping of the complete metric space $(X, d)$ into itself. Then $f$ has a fixed point in $X$ if and only if there exists $\alpha \in(0,1)$ and a mapping $g: X \rightarrow X$ which commutes with $f$ and satisfies $g(X) \subset f(X)$ and

$$
(d(g(x), g(y))) \leq \alpha d(f(x), f(y))
$$

$\forall x, y \in X$ then $f$ and $g$ have a common fixed point in $X$.

Theorem 1.3. [17] Let $f$ and $g$ be mappings of a complete metric space $X$ into itself with $f$ continuous. Let $f$ and $g$ commute with each other and $g(X) \subset f(X)$. Also, let $g$ satisfiy the following conditions:

$$
\begin{aligned}
& (d(g x, g y)) \\
& \leq \alpha_{1} d(g(x), f(x))+\alpha_{2} d(g(y), f(y)) \\
& +\alpha_{3} d(g(x), f(y))+\alpha_{4} d(g(y), f(x)) \\
& +\alpha_{5} d(f(x), f(y))
\end{aligned}
$$

with $\alpha_{i} \geq 0$ for all $i$ and $\alpha_{1}+\alpha_{2}+\alpha_{3}+2 \alpha_{4}+\alpha_{5}<1$ then $f$ and $g$ have a unique common fixed point in $X$.

Theorem 1.4. [18] Let $T$ be a mapping of the complete metric space $X$ into itself satisfying the inequality

$$
\begin{aligned}
& (d(T x, T y))^{2} \\
& \leq \alpha_{1}(d(x, T x) d(y, T y)+d(x, T y) d(y, T x)) \\
& +\alpha_{2}(d(x, T x) d(y, T x)+d(x, T y) d(y, T y))
\end{aligned}
$$

$\forall x, y \in X$ and $\alpha_{1}, \alpha_{2} \geq 0$ such that $\alpha_{1}+2 \alpha_{2}<1$ then $T$ has a unique fixed point. 
Theorem 1.5. [21] Let $T$ be a mapping of the complete metric space $X$ into itself satisfy the conditions:

$$
\begin{aligned}
& (d(T x, T y))^{2} \\
& \leq a(d(x, T x), d(y, T y)+d(x, T y), d(y, T x)) \\
& +b(d(x, T x), d(y, T x)+d(x, T y), d(y, T y)) \\
& +c\left\{[d(y, T x)]^{2}+[d(y, T y)]^{2}\right\}
\end{aligned}
$$

$\forall x, y \in X$ and $a, b, c \geq 0$ such that $a+2 b+c<1$, then $T$ has a unique fixed point in $X$.

\section{Preliminaries}

Definition 2.1. Let $(X, d)$ be a metric space. A mapping $T: X \rightarrow X$ is said to be sequentially convergent if for every sequence $\left\{y_{n}\right\}$ of $X$ if $\left\{T y_{n}\right\}$ is convergent then $\left\{y_{n}\right\}$ has a convergent subsequence.

Definition 2.2. Let $T: X \rightarrow X$ be a continuous self-map of $X$ into itself. Then $T$ is said to be contractive if $d(T x, T y)<d(x, y) \quad \forall x, y \in X, x \neq y$.

Definition 2.3. If $X$ is a non empty set and $d: X \times X \rightarrow[0, \infty)$ is a mapping satisfy the conditions:

(i) $0 \leq d(x, y) \forall x, y \in X$ and $d(x, y)=0$ if and only if $x=y$.

(ii) $d(x, y)=d(y, x) \forall x, y \in X$.

(iii) $d(x, y) \leq(d(x, z)+d(z, y)) \forall x, y, z \in X$.

Then $d$ is called a metric on $X$ and the pair $(X, d)$ is called a metric space.

Definition 2.4. A point $x \in X$ is said to be a fixed point of a self-map $T: X \rightarrow X$ if $T(x)=x$.

Definition 2.5. (i) A sequence $\left\{x_{n}\right\}$ in a metric space $(X, d)$ is said to converge to a point $x \in X$ if for every $\in>0$, there exists $N \in \mathbb{N}$ such that $d\left(x_{n}, x\right)<\in, \forall n \in N$ denoted by $\lim _{n \rightarrow \infty} x_{n}=x$.

(ii) $\left\{x_{n}\right\}$ is called Cauchy sequence if for some $N \in \mathbb{N}$ there exists $\in>0$ such that for $m, n \in N, m>n$ we have $\lim _{m, n \rightarrow \infty} d\left(x_{m}, x_{n}\right)=0$.

(iii) A metric space $(X, d)$ is said to be complete if and only if every Cauchy sequence in $X$ converges to a point of $X$.

\section{Main Results}

In this section we prove our main results on the common fixed points of commuting self-mappings on complete, compact and Hausdorff spaces. We start with the following result.

Theorem 3.1. Let $(X, d)$ be a complete metric space and let $f, g: X \rightarrow X$ be commuting self maps of $X$ into itself with $f$ continuous such that $g(X) \subset f(X)$ and $g$ satisfy the conditions

$$
\begin{aligned}
& {[d(g(x), g(y))]^{2}} \\
& \leq \alpha_{1}[d(f(x), g(x)) d(f(y), g(y))] \\
& +\alpha_{2}\left[d(f(y), g(y))^{2}(1+d(f(y), g(x)))\right] \\
& +\alpha_{3}[d(f(y), g(y)) d(g(x), g(y))(1+d(f(y), g(x)))] \\
& +\alpha_{4}[d(f(x), g(y)) d(f(y), g(y))] \\
& +\left[\begin{array}{c}
\left.\alpha_{5}\left\{\frac{(1+d(f(y), f(x))) d(f(y), g(y))}{(1+d(f(x), g(x)))}\right\}^{2}\right] \\
+\alpha_{6}\left\{\frac{d(f(y), g(x)) d(f(y), g(y))}{(1+d(f(x), g(x)))}\right\}^{2}
\end{array}\right]
\end{aligned}
$$

$\forall x, y \in X$, where $\alpha_{1}, \alpha_{2}, \alpha_{3}, \alpha_{4}, \alpha_{5}, \alpha_{6} \geq 0$ such that

$$
\alpha_{1}+\alpha_{2}+\alpha_{3}+2 \alpha_{4}+\alpha_{5}<1
$$

then $f$ and $g$ have a unique common fixed point in $X$.

Proof Let $x_{0}$ be an arbitrary point in $X$, then there exists $x_{1} \in X$ such that $g\left(x_{0}\right)=f\left(x_{1}\right)$ we construct sequence $\left\{x_{n}\right\}$ in $X$ such that $g\left(x_{n}\right)=f\left(x_{n+1}\right)$, since $g(X) \subset f(X)$ with $n=0,1,2,3, \ldots$ Now by using (3.1), we get

$$
\begin{aligned}
& {\left[d\left(g\left(x_{n}\right), g\left(x_{n+1}\right)\right)\right]^{2}} \\
& \leq \alpha_{1}\left[d\left(f\left(x_{n}\right), g\left(x_{n}\right)\right) d\left(f\left(x_{n+1}\right), g\left(x_{n+1}\right)\right)\right] \\
& +\alpha_{2}\left[d\left(f\left(x_{n+1}\right), g\left(x_{n+1}\right)\right)^{2}\left(1+d\left(f\left(x_{n+1}\right), g\left(x_{n}\right)\right)\right)\right] \\
& +\alpha_{3}\left[\begin{array}{l}
d\left(f\left(x_{n+1}\right), g\left(x_{n+1}\right)\right) d\left(g\left(x_{n}\right), g\left(x_{n+1}\right)\right) \\
\left(1+d\left(f\left(x_{n+1}\right), g\left(x_{n}\right)\right)\right)
\end{array}\right] \\
& +\alpha_{4}\left[d\left(f\left(x_{n}\right), g\left(x_{n+1}\right)\right) d\left(f\left(x_{n+1}\right), g\left(x_{n+1}\right)\right)\right] \\
& +\left[\begin{array}{l}
\alpha_{5}\left\{\frac{\left(1+d\left(f\left(x_{n+1}\right), f\left(x_{n}\right)\right)\right) d\left(f\left(x_{n+1}\right), g\left(x_{n+1}\right)\right)}{\left(1+d\left(f\left(x_{n}\right), g\left(x_{n}\right)\right)\right)}\right\}^{2} \\
+\alpha_{6}\left\{\frac{d\left(f\left(x_{n+1}\right), g\left(x_{n}\right)\right) d\left(f\left(x_{n+1}\right), g\left(x_{n+1}\right)\right)}{\left(1+d\left(f\left(x_{n}\right), g\left(x_{n}\right)\right)\right)}\right\}^{2}
\end{array}\right]
\end{aligned}
$$

or

$$
\begin{aligned}
& {\left[d\left(f\left(x_{n+1}\right), f\left(x_{n+2}\right)\right)\right]^{2}} \\
& \leq \alpha_{1}\left[d\left(f\left(x_{n}\right), f\left(x_{n+1}\right)\right) d\left(f\left(x_{n+1}\right), f\left(x_{n+2}\right)\right)\right] \\
& +\alpha_{2}\left[d\left(f\left(x_{n+1}\right), f\left(x_{n+2}\right)\right)^{2}\left(1+d\left(f\left(x_{n+1}\right), f\left(x_{n+1}\right)\right)\right)\right] \\
& +\alpha_{3}\left[\begin{array}{l}
d\left(f\left(x_{n+1}\right), f\left(x_{n+2}\right)\right) d\left(f\left(x_{n+1}\right), f\left(x_{n+2}\right)\right) \\
\left(1+d\left(f\left(x_{n+1}\right), f\left(x_{n+1}\right)\right)\right)
\end{array}\right]
\end{aligned}
$$




$$
\begin{aligned}
& +\alpha_{4}\left[d\left(f\left(x_{n}\right), f\left(x_{n+2}\right)\right) d\left(f\left(x_{n+1}\right), f\left(x_{n+2}\right)\right)\right] \\
& +\left[\begin{array}{l}
\alpha_{5}\left\{\frac{\left(1+d\left(f\left(x_{n+1}\right), f\left(x_{n}\right)\right)\right) d\left(f\left(x_{n+1}\right), f\left(x_{n+2}\right)\right)}{\left(1+d\left(f\left(x_{n}\right), f\left(x_{n+1}\right)\right)\right)}\right\}^{2} \\
+\alpha_{6}\left\{\frac{d\left(f\left(x_{n+1}\right), f\left(x_{n+1}\right)\right) d\left(f\left(x_{n+1}\right), f\left(x_{n+2}\right)\right)}{\left(1+d\left(f\left(x_{n}\right), f\left(x_{n+1}\right)\right)\right)}\right\}^{2}
\end{array}\right]
\end{aligned}
$$

or

$$
\begin{aligned}
& d\left(f\left(x_{n+1}\right), f\left(x_{n+2}\right)\right) \\
& \leq \alpha_{1} d\left(f\left(x_{n}\right), f\left(x_{n+1}\right)\right)+\alpha_{2} d\left(f\left(x_{n+1}\right), f\left(x_{n+2}\right)\right) \\
& +\alpha_{3} d\left(f\left(x_{n+1}\right), f\left(x_{n+2}\right)\right)+\alpha_{4} d\left(f\left(x_{n}\right), f\left(x_{n+2}\right)\right) \\
& +\alpha_{5} d\left(f\left(x_{n+1}\right), f\left(x_{n+2}\right)\right)
\end{aligned}
$$

or

$$
\begin{aligned}
& {\left[1-\left(\alpha_{2}+\alpha_{2}+\alpha_{3}\right)\right] d\left(f\left(x_{n+1}\right), f\left(x_{n+2}\right)\right)} \\
& \leq\left(\alpha_{1}+\alpha_{4}\right) d\left(f\left(x_{n}\right), f\left(x_{n+1}\right)\right) \\
& \Rightarrow d\left(f\left(x_{n+1}\right), f\left(x_{n+2}\right)\right) \\
& \leq \frac{\left(\alpha_{1}+\alpha_{4}\right)}{\left[1-\left(\alpha_{2}+\alpha_{4}+\alpha_{5}\right)\right]} d\left(f\left(x_{n}\right), f\left(x_{n+1}\right)\right) \\
& \Rightarrow d\left(f\left(x_{n+1}\right), f\left(x_{n+2}\right)\right) \leq \eta d\left(f\left(x_{n}\right), f\left(x_{n+1}\right)\right)
\end{aligned}
$$

where $\eta=\frac{\left(\alpha_{1}+\alpha_{4}\right)}{\left[1-\left(\alpha_{2}+\alpha_{4}+\alpha_{5}\right)\right]}<1$. On continous repeation of the above process, we get that $\forall n \in N$

$$
d\left(f\left(x_{n+1}\right), f\left(x_{n+2}\right)\right) \leq \eta^{n} d\left(f\left(x_{1}\right), f\left(x_{0}\right)\right)
$$

on taking limit as $n \rightarrow \infty$, we get

$$
\lim _{n \rightarrow \infty} d\left(f\left(x_{n+1}\right), f\left(x_{n+2}\right)\right)=0 .
$$

Since $X$ is complete, there exists $z \in X$ such that

$$
\lim _{n \rightarrow \infty} g\left(x_{n}\right)=\lim _{n \rightarrow \infty} f\left(x_{n+1}\right)=z .
$$

Since $f$ is continuous and $f, g$ commute, we have

$$
f z=f\left(\lim _{n \rightarrow \infty} f x_{n}\right)=\lim _{n \rightarrow \infty} f^{2} x_{n}
$$

also

$$
f z=f\left(\lim _{n \rightarrow \infty} g x_{n}\right)=\lim _{n \rightarrow \infty} f g x_{n}=\lim _{n \rightarrow \infty} g f x_{n} .
$$

Now by (3.1) we have

$$
\begin{aligned}
& {\left[d\left(g\left(f\left(x_{n}\right)\right), g(g(z))\right)\right]^{2}} \\
& \leq \alpha_{1}\left[d\left(f\left(f\left(x_{n}\right)\right), g\left(f\left(x_{n}\right)\right)\right) d(f(g(z)), g(g(z)))\right] \\
& +\alpha_{2}\left[\begin{array}{l}
d(f(g(z)), g(g(z)))^{2} \\
\left(1+d\left(f(g(z)), g\left(f\left(x_{n}\right)\right)\right)\right)
\end{array}\right]
\end{aligned}
$$

$$
\begin{aligned}
& +\alpha_{3}\left[\begin{array}{l}
d(f(g(z)), g(g(z))) d\left(g\left(f\left(x_{n}\right)\right), g(g(z))\right) \\
\left(1+d\left(f(g(z)), g\left(f\left(x_{n}\right)\right)\right)\right)
\end{array}\right] \\
& +\alpha_{4}\left[d\left(f\left(f\left(x_{n}\right)\right), g(g(z))\right) d(f(g(z)), g(g(z)))\right] \\
& +\left[\begin{array}{c}
\alpha_{5}\left\{\frac{\left[\begin{array}{l}
\left(1+d\left(f(g(z)), f\left(f\left(x_{n}\right)\right)\right)\right) \\
d(f(g(z)), g(g(z)))
\end{array}\right]}{\left(1+d\left(f\left(f\left(x_{n}\right)\right), g\left(f\left(x_{n}\right)\right)\right)\right)}\right\}^{2} \\
+\alpha_{6}\left\{\frac{\left[\begin{array}{l}
d\left(f(g(z)), g\left(f\left(x_{n}\right)\right)\right) \\
d(f(g(z)), g(g(z)))
\end{array}\right]}{\left(1+d\left(f\left(f\left(x_{n}\right)\right), g\left(f\left(x_{n}\right)\right)\right)\right)}\right\}
\end{array}\right\} .
\end{aligned}
$$

Now, since $f$ is continuous, we have

$$
g\left(f\left(x_{n}\right)\right)=f\left(g\left(x_{n}\right)\right)=f\left(f\left(x_{n+1}\right)\right)=f(z)
$$

which implies

$$
\begin{aligned}
& {\left[d\left(g\left(f\left(x_{n}\right)\right), g(g(z))\right)\right]^{2}} \\
& \leq \alpha_{1}\left[d\left(f\left(f\left(x_{n}\right)\right), g\left(f\left(x_{n}\right)\right)\right) d(f(g(z)), g(g(z)))\right] \\
& +\alpha_{2}\left[\begin{array}{l}
d(f(g(z)), g(g(z)))^{2} \\
\left(1+d\left(f(g(z)), g\left(f\left(x_{n}\right)\right)\right)\right)
\end{array}\right] \\
& +\alpha_{3}\left[\begin{array}{l}
d(f(g(z)), g(g(z))) d(g(f(z)), g(g(z))) \\
\left(1+d\left(f(g(z)), g\left(f\left(x_{n}\right)\right)\right)\right)
\end{array}\right] \\
& +\alpha_{4}\left[d\left(f\left(f\left(x_{n}\right)\right), g(g(z))\right) d(f(g(z)), g(g(z)))\right]
\end{aligned}
$$

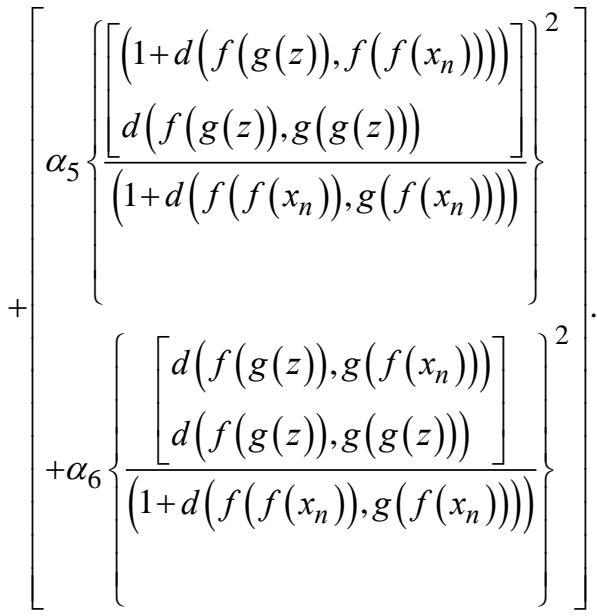

On taking lim as $n \rightarrow \infty$ and use continuity, we obtain

$$
\begin{aligned}
& {[d((f(z)), g(g(z)))]^{2}} \\
& \leq \alpha_{1}[d(f(z),(f(z))) d(f(g(z)), g(g(z)))]
\end{aligned}
$$


$+\alpha_{2}\left[d(f(g(z)), g(g(z)))^{2}(1+d(f(g(z)), f(z)))\right]$

$+\alpha_{3}\left[d(f(g(z)), g(g(z)))^{2}(1+d(f(g(z)), f(z)))\right]$

$+\alpha_{4}[d((f(z)), g(g(z))) d(f(g(z)), g(g(z)))]$

$+\alpha_{5}\left[\begin{array}{l}\left\{\frac{(1+d(f(g(z)),(f(z)))) d(f(g(z)), g(g(z)))}{(1+d((f(z)),(f(z))))}\right\}^{2} \\ +\alpha_{6}\left\{\frac{d(f(g(z)),(f(z))) d(f(g(z)), g(g(z)))}{(1+d((f(z)),(f(z))))}\right\}^{2}\end{array}\right]$.

Since $f$ and $g$ commute, we have

$$
\begin{aligned}
& {[d((f(z)), g(g(z)))]^{2}} \\
& \leq \alpha_{1}[d(f(z),(f(z))) d(f(z), g(g(z)))] \\
& +\alpha_{2}\left[d(f(z), g(g(z)))^{2}(1+d(f(z), f(z)))\right] \\
& +\alpha_{3}[d(f(z), g(g(z)))(1+d(f(z), f(z)))] \\
& +\alpha_{4}[d((f(z)), g(g(z))) d(f(z), g(g(z)))] \\
& {\left[\begin{array}{l}
\alpha_{5}\left\{\frac{(1+d(f(z),(f(z)))) d(f(z), g(g(z)))}{(1+d(f(z), f(z)))}\right\}^{2} \\
+\alpha_{6}\left\{\frac{d(f(z),(f(z))) d(f(g(z)), g(g(z)))}{(1+d(f(z), f(z)))}\right\}^{2}
\end{array}\right]} \\
& d((f(z)), g(g(z))) \\
& \leq \alpha_{2} d(f(z), g(g(z)))+\alpha_{3} d(f(z), g(g(z))) \\
& +\alpha_{4} d(f(z), g(g(z)))+\alpha_{5} d(f(z), g(g(z))) \\
& \leq\left(\alpha_{2}+\alpha_{3}+\alpha_{4}+\alpha_{5}\right) d(f(z), g(g(z))) \\
& \Rightarrow\left[1-\left(\alpha_{2}+\alpha_{3}+\alpha_{4}+\alpha_{5}\right)\right] d(f(z), g(g(z))) \leq 0 .
\end{aligned}
$$

This implies that

$$
f(z)=g(g(z)) .
$$

We now prove that $g(z)$ is a common fixed point of $f$ and g. By (3.1) we have

$$
\begin{aligned}
& {[d(g(g(z)), g(z))]^{2}} \\
& \leq \alpha_{1}[d(f(g(z)), g(g(z))) d(f(z), g(z))] \\
& +\alpha_{2}\left[d(f(z), g(z))^{2}(1+d(f(z), g(g(z))))\right] \\
& +\alpha_{3}\left[\begin{array}{l}
d(f(z), g(z)) d(g(g(z)), g(z)) \\
(1+d(f(z), g(g(z))))
\end{array}\right]
\end{aligned}
$$

$$
\begin{aligned}
& +\alpha_{4}[d(f(g(z)), g(z)) d(f(z), g(z))] \\
& +\left[\begin{array}{l}
\alpha_{5}\left\{\frac{(1+d(f(z), f(g(z)))) d(f(z), g(z))}{(1+d(f(g(z)), g(g(z))))}\right\}^{2} \\
+\alpha_{6}\left\{\frac{d(f(z), g(g(z))) d(f(z), g(z))}{(1+d(f(g(z)), g(g(z))))}\right\}^{2}
\end{array}\right]
\end{aligned}
$$

or

$$
\begin{aligned}
& {[d(f(z), g(z))]^{2}} \\
& \leq \alpha_{1}[d(f(z), f((z))) d(f(z), g(z))] \\
& +\alpha_{2}\left[d(f(z), g(z))^{2}(1+d(f(z), f(z)))\right] \\
& +\alpha_{3}\left[\begin{array}{l}
d(f(z), g(z)) d(f(z), g(z))] \\
+1+d(f(z), f(z)))
\end{array}\right] \\
& +\alpha_{4}[d(f(z), g(z)) d(f(z), g(z))] \\
& \left.+\begin{array}{l}
\alpha_{5}\left\{\frac{(1+d(f(z), f(z))) d(f(z), g(z))}{(1+d(f(z), f(z)))}\right\}^{2} \\
+\alpha_{6}\left\{\frac{d(f(z), f(z)) d(f(z), g(z))}{(1+d(f(z), f(z)))}\right\}^{2}
\end{array}\right] \\
& d(f(z), g(z)) \\
& \leq \alpha_{2} d(f(z), g(z))+\alpha_{3} d(f(z), g(z)) \\
& +\alpha_{4} d(f(z), g(z))+\alpha_{5} d(f(z), g(z)) \\
& \Rightarrow\left[1-\left(\alpha_{2}+\alpha_{3}+\alpha_{4}+\alpha_{5}\right)\right] d(f(z), g(z)) \leq 0
\end{aligned}
$$

and the inequality is possible only if

$$
d(f(z), g(z))=0 \Rightarrow f(z)=g(z) .
$$

Hence, we have

$$
f(g(z))=g(f(z))=g(g(z))=f(z)=g(z) .
$$

Uniqueness: To see that $f$ and $g$ have only one common fixed point. Suppose $z_{1}=f\left(z_{1}\right)=g\left(z_{1}\right)$ and $z_{1}=f\left(z_{1}\right)=g\left(z_{1}\right)$. By (3.1) we get

$$
\begin{aligned}
& {\left[d\left(z_{1}, z_{2}\right)\right]^{2}=\left[d\left(g\left(z_{1}\right), g\left(z_{2}\right)\right)\right]^{2}} \\
& \leq \alpha_{1}\left[d\left(f\left(z_{1}\right), g\left(z_{1}\right)\right) d\left(f\left(z_{2}\right), g\left(z_{2}\right)\right)\right] \\
& +\alpha_{2}\left[d\left(f\left(z_{2}\right), g\left(z_{2}\right)\right)^{2}\left(1+d\left(f\left(z_{2}\right), g\left(z_{1}\right)\right)\right)\right] \\
& +\alpha_{3}\left[\begin{array}{l}
\left.d\left(f\left(z_{2}\right), g\left(z_{2}\right)\right) d\left(g\left(z_{1}\right), g\left(z_{2}\right)\right)\right] \\
\left(1+d\left(f\left(z_{2}\right), g\left(z_{1}\right)\right)\right)
\end{array}\right] \\
& +\alpha_{4}\left[d\left(f\left(z_{1}\right), g\left(z_{2}\right)\right) d\left(f\left(z_{2}\right), g\left(z_{2}\right)\right)\right]
\end{aligned}
$$




$$
\begin{aligned}
& +\alpha_{5}\left\{\frac{\left(1+d\left(f\left(z_{2}\right), f\left(z_{1}\right)\right)\right) d\left(f\left(z_{2}\right), g\left(z_{2}\right)\right)}{\left(1+d\left(f\left(z_{1}\right), g\left(z_{1}\right)\right)\right\}^{2}}\right\}^{2} \\
& +\alpha_{6}\left\{\frac{d\left(f\left(z_{2}\right), g\left(z_{1}\right)\right) d\left(f\left(z_{2}\right), g\left(\left(z_{2}\right)\right)\right)}{\left(1+d\left(f\left(z_{1}\right), g\left(z_{1}\right)\right)\right)}\right\} \\
& \leq \alpha_{1}\left[d\left(z_{1}, z_{1}\right) d\left(z_{2}, z_{2}\right)\right] \\
& +\alpha_{2}\left[d\left(z_{2}, z_{2}\right)^{2}\left(1+d\left(z_{2}, z_{1}\right)\right)\right] \\
& +\alpha_{3}\left[d\left(z_{2}, z_{2}\right) d\left(z_{1}, z_{2}\right) d\left(1+d\left(z_{2}, z_{1}\right)\right)\right] \\
& +\alpha_{4}\left[d\left(z_{1}, z_{2}\right) d\left(z_{2}, z_{2}\right)\right] \\
& +\alpha_{5}\left\{\frac{\left.\left(1+d\left(z_{2}, z_{1}\right)\right) d\left(z_{2}, z_{2}\right)\right]^{2}}{\left(1+d\left(z_{1}, z_{1}\right)\right)}\right\} \\
& +\alpha_{6}\left\{\frac{d\left(z_{2}, z_{1}\right) d\left(z_{2}, z_{2}\right)}{\left(1+d\left(z_{1}, z_{1}\right)\right)}\right\}^{2} \\
& \leq 0
\end{aligned}
$$

or

$$
\Rightarrow\left[d\left(z_{1}, z_{2}\right)\right]^{2} \leq 0
$$

which is possible only if $d\left(z_{1}, z_{2}\right)=0$ and thus we have $z_{1}=z_{2}$ which shows the uniqueness of fixed point of mappings $f$ and $g$.

Corollary 3.2. Let $(X, d)$ be a complete metric space and $g: X \rightarrow X$ be a self map of $X$ into itself such that $g$ satisfy the condition

$$
\begin{aligned}
& {[d(g(x), g(y))]^{2}} \\
& \leq \alpha_{1}[d(x, g(x)) d(y, g(y))] \\
& +\alpha_{2}\left[d(y, g(y))^{2}(1+d(y, g(x)))\right] \\
& +\alpha_{3}[d(y, g(y)) d(g(x), g(y))(1+d(y, g(x)))] \\
& +\alpha_{4}[d(x, g(y)) d(y, g(y))] \\
& +\left[\begin{array}{c}
\left.\alpha_{5}\left\{\frac{(1+d(y, x)) d(y, g(y))}{(1+d(x, g(x)))}\right\}^{2}\right] \\
\left.+\alpha_{6}\left\{\frac{d(y, g(x)) d(y, g(y))}{(1+d(x, g(x)))}\right\}^{2}\right]
\end{array}\right.
\end{aligned}
$$

$\forall x, y \in X$, where $\alpha_{1}, \alpha_{2}, \alpha_{3}, \alpha_{4}, \alpha_{5}, \alpha_{6} \geq 0$ such that

$$
\alpha_{1}+\alpha_{2}+\alpha_{3}+2 \alpha_{4}+\alpha_{5}<1
$$

then $g$ has a unique fixed point in $X$.

Proof Put $f=I_{X}$ (Identity mapping) in Theorem (3.1) we get the required result.

Remark: If we put $\alpha_{6}=0$ then we get theorem (1.3) in [17]

Remark: If we put $\alpha_{2}=\alpha_{3}=\alpha_{4}=\alpha_{5}=\alpha_{6}=0$, then we get theorem (1.1) in [16]
Remark: If $\alpha_{1}=\alpha_{2}=a, \alpha_{3}=\alpha_{4}=b$ and $\alpha_{5}=\alpha_{6}=c$, we get Theorem (1.3) in [21]

Example: Let $X=\left[0, \frac{1}{3}\right]$ is a non-empty set and $d: X \times X \rightarrow R^{+}$is a metric on $X$ given by

$$
d(x, y)=|x-y| \forall x, y \in X .
$$

If $f$ and $g$ on $X$ are given by $f(x)=x^{2}, g(x)=x^{3}$. Then $f$ and $g$ commute with each other such that $f(g(x))=g(f(x))=x^{6}$ and

$$
g(X)=\left[0, \frac{1}{27}\right] \subseteq\left[0, \frac{1}{18}\right]=f(X)
$$

with 0 as the only common fixed point of $f$ and $g$.

Now we give the proof of the above Theorem(3.2) in the context of complete compact metric space.

Theorem 3.3. Let $f$ and $g$ be two mappings of a compact metric space with $f$ is continuous. Let $f$ and $g$ commute with each other such that $g(X) \subset f(X)$ and $g$ satisfy the following condition:

$$
\begin{aligned}
& {[d(g(x), g(y))]^{2}} \\
& \leq \alpha_{1}[d(f(x), g(x)) d(f(y), g(y))] \\
& +\alpha_{2}\left[d(f(y), g(y))^{2}(1+d(f(y), g(x)))\right] \\
& +\alpha_{3}\left[\begin{array}{l}
d(f(y), g(y)) d(g(x), g(y))] \\
+\alpha_{4}[d(f(x), g(y)) d(f(y), g(y))]
\end{array}\right] \\
& {\left[\begin{array}{l}
\alpha_{5}\left\{\frac{(1+d(f(y), f(x))) d(f(y), g(y))}{(1+d(f(x), g(x)))}\right\}^{2} \\
+\alpha_{6}\left\{\frac{d(f(y), g(x)) d(f(y), g(y))}{(1+d(f(x), g(x)))}\right\}^{2}
\end{array}\right]}
\end{aligned}
$$

$\forall x, y \in X, \quad x \neq y$ and $\alpha_{i} \geq 0$ with

$$
\alpha_{1}+\alpha_{2}+\alpha_{3}+2 \alpha_{4}+\alpha_{5}=1
$$

then $f$ and $g$ have a unique common fixed point in $X$.

Proof Let $x_{0}$ be an arbitrary point of $X$. Since $g(X) \subset f(X)$, there exists $x_{1} \in X$ such that $g x_{0}=f x_{1}$. Continuing this process we get a sequence $\left\{x_{n}\right\}$ in $X$ such that

$$
g x_{n}=f x_{n+1} \forall N \bigcup\{0\} .
$$

Now from (3.4) we get

$$
\begin{aligned}
& {\left[d\left(f\left(x_{n+1}\right), f\left(x_{n+2}\right)\right)\right]^{2}=\left[d\left(g\left(x_{n}\right), g\left(x_{n+1}\right)\right)\right]^{2}} \\
& \leq \alpha_{1}\left[d\left(f\left(x_{n}\right), g\left(x_{n}\right)\right) d\left(f\left(x_{n+1}\right), g\left(x_{n+1}\right)\right)\right] \\
& +\alpha_{2}\left[d\left(f\left(x_{n+1}\right), g\left(x_{n+1}\right)\right)^{2}\left(1+d\left(f\left(x_{n+1}\right), g\left(x_{n}\right)\right)\right)\right]
\end{aligned}
$$




$$
\begin{aligned}
& +\alpha_{3}\left[\begin{array}{l}
d\left(f\left(x_{n+1}\right), g\left(x_{n+1}\right)\right) d\left(g\left(x_{n}\right), g\left(x_{n+1}\right)\right) \\
\left(1+d\left(f\left(x_{n+1}\right), g\left(x_{n}\right)\right)\right)
\end{array}\right] \\
& +\alpha_{4}\left[d\left(f\left(x_{n}\right), g\left(x_{n+1}\right)\right) d\left(f\left(x_{n+1}\right), g\left(x_{n+1}\right)\right)\right] \\
& +\left[\begin{array}{l}
\alpha_{5}\left\{\frac{\left(1+d\left(f\left(x_{n+1}\right), f\left(x_{n}\right)\right)\right) d\left(f\left(x_{n+1}\right), g\left(x_{n+1}\right)\right)}{\left(1+d\left(f\left(x_{n}\right), g\left(x_{n}\right)\right)\right)}\right\}^{2} \\
+\alpha_{6}\left\{\frac{d\left(f\left(x_{n+1}\right), g\left(x_{n}\right)\right) d\left(f\left(x_{n+1}\right), g\left(x_{n+1}\right)\right)}{\left(1+d\left(f\left(x_{n}\right), g\left(x_{n}\right)\right)\right)}\right\}^{2}
\end{array}\right] \\
& \leq \alpha_{1}\left[d\left(f\left(x_{n}\right), f\left(x_{n+1}\right)\right) d\left(f\left(x_{n+1}\right), f\left(x_{n+2}\right)\right)\right] \\
& +\alpha_{2}\left[d\left(f\left(x_{n+1}\right), f\left(x_{n+2}\right)\right)^{2}\left(1+d\left(f\left(x_{n+1}\right), f\left(x_{n+1}\right)\right)\right)\right] \\
& +\alpha_{3}\left[\begin{array}{l}
d\left(f\left(x_{n+1}\right), f\left(x_{n+2}\right)\right) d\left(f\left(x_{n+1}\right), f\left(x_{n+2}\right)\right) \\
\left(1+d\left(f\left(x_{n+1}\right), f\left(x_{n+1}\right)\right)\right)
\end{array}\right] \\
& +\alpha_{4}\left[d\left(f\left(x_{n}\right), f\left(x_{n+2}\right)\right) d\left(f\left(x_{n+1}\right), f\left(x_{n+2}\right)\right)\right] \\
& +\left[\begin{array}{l}
\alpha_{5}\left\{\frac{\left(1+d\left(f\left(x_{n+1}\right), f\left(x_{n}\right)\right)\right) d\left(f\left(x_{n+1}\right), f\left(x_{n+2}\right)\right)}{\left(1+d\left(f\left(x_{n}\right), f\left(x_{n+1}\right)\right)\right)}\right\}^{2} \\
+\alpha_{6}\left\{\frac{d\left(f\left(x_{n+1}\right), f\left(x_{n+1}\right)\right) d\left(f\left(x_{n+1}\right), f\left(x_{n+2}\right)\right)}{\left(1+d\left(f\left(x_{n}\right), f\left(x_{n+1}\right)\right)\right)}\right\}^{2}
\end{array}\right]
\end{aligned}
$$

or

$$
\begin{aligned}
& d\left(f\left(x_{n+1}\right), f\left(x_{n+2}\right)\right) \\
& \leq \alpha_{1} d\left(f\left(x_{n}\right), f\left(x_{n+1}\right)\right)+\alpha_{2} d\left(f\left(x_{n+1}\right), f\left(x_{n+2}\right)\right) \\
& +\alpha_{3} d\left(f\left(x_{n+1}\right), f\left(x_{n+2}\right)\right)+\alpha_{4} d\left(f\left(x_{n}\right), f\left(x_{n+2}\right)\right) \\
& +\alpha_{5} d\left(f\left(x_{n+1}\right), f\left(x_{n+2}\right)\right) \\
& \leq\left(\alpha_{1}+\alpha_{4}\right) d\left(f\left(x_{n}\right), f\left(x_{n+1}\right)\right) \\
& +\left(\alpha_{2}+\alpha_{3}+\alpha_{4}+\alpha_{5}\right) d\left(f\left(x_{n+1}\right), f\left(x_{n+2}\right)\right) \\
& \Rightarrow\left[1-\left(\alpha_{2}+\alpha_{3}+\alpha_{4}+\alpha_{5}\right)\right] d\left(f\left(x_{n+1}\right), f\left(x_{n+2}\right)\right) \\
& \leq\left(\alpha_{1}+\alpha_{4}\right) d\left(f\left(x_{n}\right), f\left(x_{n+1}\right)\right)
\end{aligned}
$$

or

$$
\begin{aligned}
& d\left(f\left(x_{n+1}\right), f\left(x_{n+2}\right)\right) \\
& \leq \frac{\left(\alpha_{1}+\alpha_{4}\right)}{\left[1-\left(\alpha_{2}+\alpha_{3}+\alpha_{4}+\alpha_{5}\right)\right]} d\left(f\left(x_{n}\right), f\left(x_{n+1}\right)\right) \\
& \Rightarrow d\left(f\left(x_{n+1}\right), f\left(x_{n+2}\right)\right) \leq d\left(f\left(x_{n}\right), f\left(x_{n+1}\right)\right) \forall n \geq 0 \\
& d_{n+1} \leq d_{n}, d_{n}=d\left(f\left(x_{n}\right), f\left(x_{n+1}\right)\right), \\
& d_{n+1}=d\left(f\left(x_{n+1}\right), f\left(x_{n+2}\right)\right)
\end{aligned}
$$

and $\alpha_{1}+\alpha_{2}+\alpha_{3}+2 \alpha_{4}+\alpha_{5}=1$. Hence the sequence $d_{n}=\left\{d\left(f\left(x_{n}\right), f\left(x_{n+1}\right)\right)\right\} \quad$ is a monotonic decreasing sequence of non-negative real number and so converges to a limit $d \geq 0$ such that

$$
\lim _{n \rightarrow \infty} d_{n}=\lim _{n \rightarrow \infty}\left\{d\left(f\left(x_{n}\right), f\left(x_{n+1}\right)\right)\right\}=d .
$$

Since $X$ is compact, using sequential compactness of $X$, there exists a subsequence $\left\{x_{n_{k}}\right\}$ of $\left\{x_{n}\right\}$ such that for any $u \in X$ and $k \rightarrow \infty$, we get

$$
\lim _{k \rightarrow \infty} x_{n_{k}}=u \text {. }
$$

Now, we use the continuity of $g$ and $d$ to obtain

$$
\begin{aligned}
& d_{n_{k}}=d\left(x_{n_{k}}, x_{n_{k+1}}\right) \\
& =d\left(x_{n_{k}}, g x_{n_{k}}\right) \rightarrow d(u, g u) \text { as } k \rightarrow \infty .
\end{aligned}
$$

Since $d_{n_{k}} \rightarrow d$, we have $d=d(u, g u)$. Similarly,

$$
\begin{aligned}
& d_{n_{k+1}}=d\left(x_{n_{k+1}}, x_{n_{k+2}}\right) \\
& =d\left(g x_{n_{k}}, g g x_{n_{k}}\right) \rightarrow d(g u, g g u)=d \text { as } k \rightarrow \infty .
\end{aligned}
$$

Since the sequence $\left\{d_{n_{k+1}}\right\}$ is a subsequence of the sequence $\left\{d_{n}\right\}$, we get

$$
d=d(u, g u)=d(g u, g g u) .
$$

Next we claim $d=0$. Suppose $d \neq 0$, then $u \neq g u$. By (3.6) we obtain

$$
\begin{aligned}
& d=\lim _{k \rightarrow \infty} d_{n_{k+1}}=\lim _{k \rightarrow \infty} d\left(g\left(x_{n_{k}}\right), g\left(g\left(x_{n_{k}}\right)\right)\right) \\
& =d(g(u), g(g(u)))<d(u, g(u))=d
\end{aligned}
$$

which is contradiction, hence $d=0$. Hence,

$$
\lim _{n \rightarrow \infty} d\left(f\left(x_{n}\right), f\left(x_{n+1}\right)\right)=0 .
$$

Since $g x_{n}=f x_{n+1}$ for each $n=0,1,2, \ldots$. From (3.7), we get

$$
\inf \{d(f(x), g(x)): x \in X\}=0 \text {. }
$$

Since the mapping $d: X \rightarrow R^{+}$defined by $x \rightarrow d(f(x), g(x))$ is continuous, there exists $z \in X$ such that

$$
d(f(z), g(z))=\inf \{d(f(x), g(x)): x \in X\} .
$$

By (3.8) $d(f(z), g(z))=0$ and so $f(z)=g(z)=v$. Now, since $f$ and $g$ commute, we have

$$
f(v)=f(g(z))=g(f(z))=g(v) .
$$

Thus

$$
f(v)=g(v)=v
$$

and so $v$ is a common fixed point of $f$ and $g$.

Uniqueness: Next we claim that $v$ is the unique common fixed point of $f$ and $g$. Suppose on the contrary that there exists another point $w \in X$ such that $f(w)=g(w)=w$ with $f(w) \neq f(v)$. Using condition (3.4) we get 


$$
\begin{aligned}
& {[d(v, w)]^{2}=[d(g(v), g(w))]^{2}} \\
& \leq \alpha_{1}[d(f(v), g(v)) d(f(w), g(w))] \\
& +\alpha_{2}\left[d(f(w), g(w))^{2}(1+d(f(w), g(v)))\right] \\
& +\alpha_{3}\left[\begin{array}{l}
d(f(w), g(w)) d(g(v), g(w)) \\
(1+d(f(w), g(v)))
\end{array}\right] \\
& +\alpha_{4}[d(f(v), g(w)) d(f(w), g(w))] \\
& +\left[\begin{array}{l}
\alpha_{5}\left\{\frac{(1+d(f(w), f(v))) d(f(w), g(w))}{(1+d(f(v), g(v)))}\right\}^{2} \\
+\alpha_{6}\left\{\frac{d(f(w), g(v)) d(f(w), g(w))}{(1+d(f(v), g(v)))}\right\}^{2}
\end{array}\right] \\
& \leq \alpha_{1}[d(v, v) d(w, w)]+\alpha_{2}\left[d(w, w)^{2}(1+d(w, v))\right] \\
& +\alpha_{3}[d(w, w) d(v, w)(1+d(w, v))] \\
& +\alpha_{4}[d(v, w) d(w, w)] \\
& +\left[\begin{array}{l}
\alpha_{5}\left\{\frac{(1+d(w, v)) d(w, w)}{(1+d(v, v))}\right\}^{2} \\
+\alpha_{6}\left\{\frac{d(w, v) d(w, w)}{(1+d(v, v))}\right\}^{2}
\end{array}\right] \\
& \leq 0 \\
& \Rightarrow(d(v, w))^{2} \leq 0
\end{aligned}
$$

which implies that $d(v, w)=0$ or $v=w$ and this gives the uniqueness of the fixed point.

Corollary 3.4. Let $(X, d)$ be a compact metric space and let $f, g: X \rightarrow X$ be self mappings of $X$ satisfying the condition

$$
\begin{aligned}
& {[d(g(x), g(y))]^{2}} \\
& \leq a\left[\begin{array}{l}
d(f(x), g(x)) d(f(y), g(y)) \\
+d(f(y), g(y))^{2}(1+d(f(y), g(x)))
\end{array}\right] \\
& +b\left[\begin{array}{l}
d(f(y), g(y)) d(g(x), g(y)) \\
(1+(f(y), g(x))) \\
+d(f(x), g(y)) d(f(y), g(y))
\end{array}\right] \\
& +c\left[\begin{array}{l}
\left\{\frac{(1+d(f(y), f(x))) d(f(y), g(y))}{(1+d(f(x), g(x)))}\right. \\
+\left\{\frac{d(f(y), g(x)) d(f(y), g(y))}{(1+d(f(x), g(x)))}\right\}^{2}
\end{array}\right]
\end{aligned}
$$

$\forall x, y \in X$, where $a, b, c \geq 0$ with $a+2 b+c=1$, then $f$ and $g$ have unique common fixed point in $X$.
Proof Putting $\alpha_{1}=\alpha_{2}=a, \quad \alpha_{3}=\alpha_{4}=b$, and $\alpha_{5}=c$ in Theorem (3.3) we get the required result.

Corollary 3.5. Let $(X, d)$ be a complete compact metric space and $g$ be a self mapping $g: X \rightarrow X$ satisfying

$$
\begin{aligned}
& {[d(g(x), g(y))]^{2}} \\
& \leq \alpha_{1}[d(x, g(x)) d(y, g(y))] \\
& +\alpha_{2}\left[d(y, g(y))^{2}(1+d(y, g(x)))\right] \\
& +\alpha_{3}[d(y, g(y)) d(g(x), g(y))(1+d(y, g(x)))] \\
& +\alpha_{4}[d(x, g(y)) d(y, g(y))]
\end{aligned}
$$

for all $x, y \in X$ and $\alpha_{1}, \alpha_{2}, \alpha_{3}, \alpha_{4} \geq 0$ such that $\alpha_{1}+\alpha_{2}+\alpha_{3}+2 \alpha_{4}=1$, then $\mathrm{g}$ has a unique fixed point in $X$.

Proof Putting $\alpha_{5}=0$ and $f=I$ (Identity mapping) in Theorem (3.8), we get the required result.

Corollary 3.6. Let $(X, d)$ be a compact metric space and $g: X \rightarrow X$ be a self map of $X$ into itself satisfying the inequality

$$
\begin{aligned}
& {[d(g(x), g(y))]^{2}} \\
& \leq \alpha_{1}[d(x, g(x)) d(y, g(y))] \\
& +\alpha_{2}\left[d(y, g(y))^{2}(1+d(y, g(x)))\right]
\end{aligned}
$$

for all $x, y \in X$, such that $\alpha_{1}+2 \alpha_{2}=1$, then $g$ has a unique fixed point in $X$.

Proof Putting $\alpha_{3}=\alpha_{4}=\alpha_{5}=\alpha_{6}=0$ in Theorem (3.8) we get the required result

Remark: Corollary (3.4) is the result of Sahu and Sharma in [21]

Remark: Corollary (3.5) is the result of Pachpatte [18]

Remark: Corollary (3.6) is the result of Fisher [9]

Now we give an example to support the validity of the above theorem (3.3).

Example: Let $X=\{1,5,9\}$ be any non-empty set and let $d$ be the metric with ordinary distance. Let the functions $f$ and $g$ on $X$ be defined by

$$
\begin{aligned}
& f(1)=5, f(5)=1, f(9)=9 \\
& \text { and } g(1)=g(5)=g(9)=9 .
\end{aligned}
$$

Then it is clear that $g(X) \subset f(X)$ with $f$ and $g$ commute, continuous and $(X, d)$ is a compact metric space. Now it is easy to show that the above example satisfy all the conditions of Theorem (3.3) with 9 as the only common fixed point in $X$.

Theorem 3.7. Let $f$ and $g$ be two continuous mappings of a hausdorff space $X$ into itself and let $f, g$ commute with each other such that $g(X) \subset f(X)$. Let $F: X \times X \rightarrow R^{+}$ be a continuous function such that for each pair of $x, y \in X$ with $f(x) \neq f(y)$ 


$$
\begin{aligned}
& {[F(g(x), g(y))]^{2}} \\
& \leq \alpha_{1}[F(f(x), g(x)) F(f(y), g(y))] \\
& +\alpha_{2}\left[(F(f(x), g(x)))^{2}(1+F(f(y), g(x)))\right] \\
& +\alpha_{3}\left[\begin{array}{l}
F(f(y), g(y)) F(g(x), g(y)) \\
(1+F(f(y), g(x)))
\end{array}\right] \\
& +\alpha_{4}[F(f(x), g(y)) F(f(y), g(y))] \\
& +\alpha_{5}\left\{\frac{(1+F(f(y), f(x))) F(f(y), g(y))}{1+F(f(x), g(x))}\right\}^{2} \\
& +\alpha_{6}\left\{\frac{(F(f(y), g(x))) F(f(y), g(y))}{1+F(f(x), g(x))}\right\}^{2}
\end{aligned}
$$

and $\alpha_{i} \geq 0$ such that $\alpha_{1}+\alpha_{2}+\alpha_{3}+2 \alpha_{4}+\alpha_{5}<1$. If for some $x_{0} \in X$, the sequence $\left\{x_{n}\right\}$ in $\mathrm{X}$ has a convergent subsequence. Then $f, g$ have a common fixed point.

Proof Since $g(X) \subset f(X)$. So, for $x_{0} \in X$, we choose $x_{1} \in X$ such that $g\left(x_{0}\right)=f\left(x_{1}\right)$ with the sequence $\left\{x_{n}\right\}$ defined by

$$
\begin{aligned}
& g x_{0}=f x_{1}, g x_{1}=f x_{2}, \ldots, g x_{n-1}=f x_{n}, \\
& g x_{n}=f x_{n+1}, n=0,1,2,3, \ldots
\end{aligned}
$$

Now by (3.10) we have

$$
\begin{aligned}
& {\left[F\left(f\left(x_{n+1}\right), f\left(x_{n+2}\right)\right)\right]^{2}} \\
& =\left[F\left(g\left(x_{n}\right), g\left(x_{n+1}\right)\right)\right]^{2} \\
& \leq \alpha_{1}\left[F\left(f\left(x_{n}\right), g\left(x_{n}\right)\right) F\left(f\left(x_{n+1}\right), g\left(x_{n+1}\right)\right)\right] \\
& +\alpha_{2}\left[\begin{array}{l}
\left(F\left(f\left(x_{n}\right), g\left(x_{n+1}\right)\right)\right)^{2} \\
\left(1+F\left(f\left(x_{n+1}\right), g\left(x_{n}\right)\right)\right)
\end{array}\right] \\
& +\alpha_{3}\left[\begin{array}{l}
F\left(f\left(x_{n+1}\right), g\left(x_{n+1}\right)\right) F\left(g\left(x_{n}\right), g\left(x_{n+1}\right)\right) \\
\left(1+F\left(f\left(x_{n+1}\right), g\left(x_{n}\right)\right)\right)
\end{array}\right] \\
& +\alpha_{4}\left[F\left(f\left(x_{n}\right), g\left(x_{n+1}\right)\right) F\left(f\left(x_{n+1}\right), g\left(x_{n+1}\right)\right)\right] \\
& +\alpha_{5}\left\{\frac{\left(1+F\left(f\left(x_{n+1}\right), f\left(x_{n}\right)\right)\right) F\left(f\left(x_{n+1}\right), g\left(x_{n+1}\right)\right)}{1+F\left(f\left(x_{n}\right), g\left(x_{n}\right)\right)}\right\}^{2} \\
& +\alpha_{6}\left\{\frac{F\left(f\left(x_{n+1}\right), g\left(x_{n}\right)\right) F\left(f\left(x_{n+1}\right), f\left(x_{n+1}\right)\right)}{1+F\left(f\left(x_{n}\right), g\left(x_{n}\right)\right)}\right\}^{2} \\
& \leq \alpha_{1}\left[F\left(f\left(x_{n}\right), f\left(x_{n+1}\right)\right) F\left(f\left(x_{n+1}\right), f\left(x_{n+2}\right)\right)\right] \\
& +\alpha_{2}\left[\begin{array}{l}
\left(F\left(f\left(x_{n+1}\right), f\left(x_{n+2}\right)\right)\right)^{2} \\
\left(1+F\left(f\left(x_{n+1}\right), f\left(x_{n+1}\right)\right)\right)
\end{array}\right] \\
& +\alpha_{3}\left[\begin{array}{l}
F\left(f\left(x_{n+1}\right), f\left(x_{n+2}\right)\right) F\left(f\left(x_{n+1}\right), f\left(x_{n+2}\right)\right) \\
\left(1+F\left(f\left(x_{n+1}\right), f\left(x_{n+1}\right)\right)\right)
\end{array}\right]
\end{aligned}
$$

$$
\begin{aligned}
& +\alpha_{4}\left[F\left(f\left(x_{n}\right), f\left(x_{n+2}\right)\right) F\left(f\left(x_{n+1}\right), f\left(x_{n+2}\right)\right)\right] \\
& +\alpha_{5}\left\{\frac{F\left(f\left(x_{n+1}\right), f\left(x_{n}\right)\right) F\left(f\left(x_{n+1}\right), f\left(x_{n+2}\right)\right)}{1+F\left(f\left(x_{n}\right), f\left(x_{n+1}\right)\right)}\right\}^{2} \\
& +\alpha_{6}\left\{\frac{F\left(f\left(x_{n+1}\right), f\left(x_{n+1}\right)\right) F\left(f\left(x_{n+1}\right), f\left(x_{n+2}\right)\right)}{1+F\left(f\left(x_{n}\right), f\left(x_{n+1}\right)\right)}\right\}^{2} \\
& \Rightarrow F\left(f\left(x_{n+1}\right), f\left(x_{n+2}\right)\right) \leq \alpha_{1} F\left(f\left(x_{n}\right), f\left(x_{n+1}\right)\right) \\
& +\alpha_{2} F\left(f\left(x_{n+1}\right), f\left(x_{n+2}\right)\right)+\alpha_{3} F\left(f\left(x_{n+1}\right), f\left(x_{n+2}\right)\right) \\
& +\alpha_{4} F\left(f\left(x_{n}\right), f\left(x_{n+2}\right)\right)+\alpha_{5} F\left(f\left(x_{n+1}\right), f\left(x_{n+2}\right)\right)
\end{aligned}
$$

or

$$
\begin{aligned}
& F\left(f\left(x_{n+1}\right), f\left(x_{n+2}\right)\right) \\
& \leq \frac{\left(\alpha_{1}+\alpha_{4}\right)}{\left[1-\left(\alpha_{2}+\alpha_{3}+\alpha_{4}+\alpha_{5}\right)\right]} F\left(f\left(x_{n}\right), f\left(x_{n+1}\right)\right) .
\end{aligned}
$$

Let $y_{n}=f\left(x_{n}\right) \forall n \in N$. Then (3.11) gives us

$$
\begin{aligned}
& F\left(y_{1}, y_{2}\right)=F\left(f\left(x_{1}\right), f\left(x_{2}\right)\right) \\
& \leq \frac{\left(\alpha_{1}+\alpha_{4}\right)}{\left[1-\left(\alpha_{2}+\alpha_{3}+\alpha_{4}+\alpha_{5}\right)\right]} F\left(f\left(x_{0}\right), f\left(x_{1}\right)\right) \\
& <F\left(y_{0}, y_{1}\right) .
\end{aligned}
$$

Similarly,

$$
\begin{aligned}
& F\left(y_{2}, y_{3}\right)=F\left(f\left(x_{2}\right), f\left(x_{3}\right)\right) \\
& \leq \frac{\left(\alpha_{1}+\alpha_{4}\right)}{\left[1-\left(\alpha_{2}+\alpha_{3}+\alpha_{4}+\alpha_{5}\right)\right]} F\left(f\left(x_{1}\right), f\left(x_{2}\right)\right) \\
& <F\left(y_{1}, y_{2}\right) .
\end{aligned}
$$

Since $\alpha_{1}+\alpha_{2}+\alpha_{3}+2 \alpha_{4}+\alpha_{5}<1$.

Repeating the above process, we get

$$
F\left(y_{0}, y_{1}\right) \geq F\left(y_{2}, y_{3}\right) \geq \ldots \geq F\left(y_{n}, y_{n+1}\right) \geq \ldots
$$

This shows that the sequence $F\left(y_{n}, y_{n+1}\right)$ is bounded which converges along with all its subsequences to some positive real number $z$. If $\left\{y_{n}\right\}$ has a convergent subsequence of $\left\{y_{n_{k}}\right\}$ which converges to the real number $z$. Then,

$$
\begin{aligned}
& F(z, g(z))=F\left[\lim _{k \rightarrow \infty}\left(y_{n_{k}}\right), g\left(\lim _{k \rightarrow \infty}\left(y_{n_{k}}\right)\right)\right] \\
& =F\left[\lim _{k \rightarrow \infty}\left(y_{n_{k}}\right), g\left(\lim _{k \rightarrow \infty}\left(y_{n_{k+1}}\right)\right)\right] \\
& =F \lim _{k \rightarrow \infty}\left[\left\{y_{n_{k+1}}\right\},\left\{y_{n_{k+2}}\right\}\right] \\
& =F\left[\lim _{k \rightarrow \infty}\left(y_{n_{k+1}}\right), \lim _{k \rightarrow \infty}\left(y_{n_{k+2}}\right)\right] \\
& =F\left[g \lim _{k \rightarrow \infty}\left(y_{n_{k}}\right), g\left(g \lim _{k \rightarrow \infty}\left(y_{n_{k}}\right)\right)\right] \\
& =F(g(z), g(g(z))) .
\end{aligned}
$$


Next we show that $z$ is a fixed point of $f$ and $g$. First we show that $z$ is a fixed point of $g$. Suppose, $g(z) \neq z$, then by (3.5) we have

$$
\begin{aligned}
& {[F(z, g(z))]^{2}=[F(g(z), g(g(z)))]^{2}} \\
& \leq \alpha_{1}[F(f(z), g(z)) F(f(g(z)), g(g(z)))] \\
& +\alpha_{2}\left[(F(f(g(z)), g(g(z))))^{2}(1+F(f(g(z)), g(z)))\right] \\
& +\alpha_{3}\left[\begin{array}{l}
F(f(g(z)), g(g(z))) F(g(z), g(g(z))) \\
(1+F(f(g(z)), g(z)))
\end{array}\right] \\
& +\alpha_{4}[F(f(z), g(g(z))) F(f(g(z)), g(g(z)))] \\
& +\alpha_{5}\left\{\frac{(1+F(f(g(z)), f(z))) F(f(g(z)), g(g(z)))}{1+F(f(z), g(z))}\right\}^{2} \\
& +\alpha_{6}\left\{\frac{(F(f(g(z)), g(z))) F(f(g(z)), g(g(z)))}{1+F(f(z), g(z))}\right\}^{2} \\
& \leq \alpha_{1}[F(g(z), g(z)) F((g(z)), g(g(z)))] \\
& +\alpha_{2}\left[(F((g(z)), g(g(z))))^{2}(1+F((g(z)), g(z)))\right] \\
& +\alpha_{3}\left[\begin{array}{l}
F((g(z)), g(g(z))) F(g(z), g(g(z))) \\
(1+F((g(z)), g(z)))
\end{array}\right] \\
& +\alpha_{4}[F(g(z), g(g(z))) F((g(z)), g(g(z)))] \\
& +\alpha_{5}\left\{\frac{(1+F((g(z)), g(z))) F((g(z)), g(g(z)))}{1+F(g(z), g(z))}\right\}^{2} \\
& +\alpha_{6}\left\{\frac{(F((g(z)), g(z))) F((g(z)), g(g(z)))}{1+F(f(z), g(z))}\right\}^{2} \\
& \leq \alpha_{1}[F(z, z) F(z, g(z))] \\
& +\alpha_{2}\left[(F(z, g(z)))^{2}(1+F(z, z))\right] \\
& +\alpha_{3}[F(z, g(z)) F(z, g(z))(1+F(z, z))] \\
& +\alpha_{4}[F(z, g(z)) F(z, g(z))] \\
& +\alpha_{5}(F(z, g(z)))^{2}
\end{aligned}
$$

or

$$
\begin{aligned}
& F(z, g(z)) \\
& \leq \alpha_{2} F(z, g(z))+\alpha_{3} F(z, g(z)) \\
& +\alpha_{4} F(z, g(z))+\alpha_{5} F(z, g(z)) \\
& \Rightarrow\left[1-\left(\alpha_{2}+\alpha_{3}+\alpha_{4}+\alpha_{5}\right)\right] F(z, g(z)) \leq 0
\end{aligned}
$$

which is contradiction because $\alpha_{1}+\alpha_{2}+\alpha_{3}+2 \alpha_{4}+\alpha_{5}<1$. Hence, $F(z, g(z))=0 \Rightarrow z=g(z)$. Thus, $z \in X$ is a fixed point of $g$. Since $f$ and $g$ commute and are continuous, we have

$$
\begin{aligned}
& f\left(g\left(y_{n_{k}}\right)\right) \rightarrow f(z) \\
& \text { and } g\left(f\left(y_{n_{k}}\right)\right) \rightarrow g(z) \text { as } k \rightarrow \infty \\
& f\left(g\left(y_{n_{k}}\right)\right)=g\left(f\left(y_{n_{k}}\right)\right) \text { as } n_{k} \rightarrow \infty .
\end{aligned}
$$

By the uniqueness of limit we have

$$
f(z)=g(z)=z .
$$

Uniqueness: Now we claim that $z$ is the unique common fixed point of $f$ and $g$. Suppose for contradiction that $w$ is another fixed point of $f$ and $g$ such that $f(w)=g(w)=w$ then by (3.10) we obtain

$$
\begin{aligned}
& {[F(z, w)]^{2}=[F(g(z), g(w))]^{2}} \\
& \leq \alpha_{1}[(F(f(z), g(z))) F(f(w), g(w))] \\
& +\alpha_{2}\left[(F(f(w), g(w)))^{2}(1+F(f(w), g(z)))\right] \\
& +\alpha_{3}\left[\begin{array}{l}
F(f(w), g(w)) F(g(z), g(w))] \\
+\alpha_{4}[F(f(w), g(z)))
\end{array}\right] \\
& +\alpha_{5}\left\{\frac{(1+F(f(w), g(w))(f(w), g(w))]}{1+F(f(z))) F(f(z))}\right\} \\
& +\alpha_{6}\left\{\frac{(F(f(w), g(z))) F(f(w), g(w))}{1+F(f(z), g(z))}\right)^{2} \\
& \leq \alpha_{1}[(F(z, z)) F(w, w)] \\
& +\alpha_{2}\left[(F(w, w))^{2}(1+F(w, z))\right] \\
& +\alpha_{3}[F(w, w) F(z, w)(1+F(w, z))] \\
& +\alpha_{5}\left\{\frac{(1+F(w, z)) F(w, w))^{2}}{1+F(z, z)}\right\} \\
& \leq 0 .
\end{aligned}
$$

This implies that $[F(z, w)]^{2} \leq 0$ which is contradiction because

$$
\alpha_{1}+\alpha_{2}+\alpha_{3}+2 \alpha_{4}+\alpha_{5}<1
$$

Hence, $[F(z, w)]^{2}=0 \Rightarrow z=w$. Hence $z$ is the unique common fixed point of $f$ and $g$.

Finally, we provide example to check the validity of Theorem (3.7).

Corollary 3.8. Let $g: X \rightarrow X$ be a continuous mappings of a hausdorff space $\mathrm{X}$ into itself and let $F: X \times X \rightarrow R^{+}$ 
be a continuous function such that for each pair of $x, y \in X$ with $f(x) \neq f(y)$

$$
\begin{aligned}
& {[F(g(x), g(y))]^{2}} \\
& \leq \alpha_{1}[(F(x, g(x))) F(y, g(y))] \\
& +\alpha_{2}\left[(F(y, g(y)))^{2}(1+F(y, g(x)))\right] \\
& +\alpha_{3}[F(y, g(y)) F(g(x), g(y))(1+F(y, g(x)))] \\
& +\alpha_{4}[F(x, g(y))(y, g(y))] \\
& +\alpha_{5}\left\{\frac{(1+F(y, x)) F(y, g(y))}{1+F(x, g(x))}\right\}^{2} \\
& +\alpha_{6}\left\{\frac{(F(y, g(x))) F(y, g(y))}{1+F(x, g(x))}\right\}^{2}
\end{aligned}
$$

and $\alpha_{i} \geq 0$ such that

$$
\alpha_{1}+\alpha_{2}+\alpha_{3}+2 \alpha_{4}+\alpha_{5}<1
$$

If for some $x_{0} \in X$, the sequence $\left\{x_{n}\right\}$ in $X$ has a convergent subsequence. Then $f$ has a unique fixed point in $X$.

Proof Put $f=I_{X}$ (Identity mapping) in Theorem (3.7) we get the required result.

Corollary 3.9. Let $f$ and g be two continuous mappings of a hausdorff space $X$ into itself and let $f, g$ commute with each other such that $g(X) \subset f(X)$. Let $F: X \times X \rightarrow R^{+}$ be a continuous function such that for each pair of $x, y \in X$ with $f(x) \neq f(y)$

$$
\begin{aligned}
& {[F(g(x), g(y))]^{2}} \\
& \leq \alpha_{1}[(F(f(x), g(x))) F(f(y), g(y))] \\
& +\alpha_{2}\left[(F(f(y), g(y)))^{2}(1+F(f(y), g(x)))\right]
\end{aligned}
$$

and $\alpha_{1}, \alpha_{2} \geq 0$ such that $\alpha_{1}+\alpha_{2}<1$. If for some $x_{0} \in X$, the sequence $\left\{x_{n}\right\}$ in $X$ has a convergent subsequence. Then $f, g$ have a common fixed point.

Proof Put $\alpha_{3}=\alpha_{4}=\alpha_{5}=\alpha_{6}=0$ in Theorem (3.7) we get Corollary (3.9).

Remark: Corollary (3.8) is the result of [17]

Remark: Corollary (3.9) is the result of [5]

Example: Let $X=\{3,4,5\}$. We define $F: X \times X \rightarrow[0, \infty)$ and $f, g: X \rightarrow X$ by

$$
\begin{aligned}
& F(x, x)=0 \text { and } F(x, y)=F(y, x) \forall x, y \in X \\
& \text { with } F(3,4)=1, F(3,5)=F(4,5)=2, \\
& \text { and } g(3)=g(4)=g(5)=5 .
\end{aligned}
$$

Now, it is clear that the conditions of Theorem (3.7) are satisfied and that 5 is the common fixed point of $f$ and $g$.

\section{References}

[1] Bailey D. F. Some theorems on contractive mappings, J. Londdon. Math. Soc., 101-106, 2(1966).

[2] Banach S. Sur les operation dans les ensembles abstraits etleur application aux equations integrals Fund. Math. 3: 133-181(1922).

[3] Bondar K.L. Some fixed point theorems for contractive type mappings on Hausdorff space International Mathematical Forum Vol.6, no.49, 2403-2408 (2011).

[4] Browder B.E. Remarks on fixed point theorems of contractive type Nonlinear Anal. T. M. A., 3, 657-661 (1979).

[5] Chatterjee H. On generalization of Banach contraction principle, Indian J.pure. appl. Math., 10, 400-403 (1979).

[6] Ciric Lj. B A generalization of Banach.s Contraction principle Proc. Amer. Math. Soc., 45, 267-274 (1974).

[7] Edelstein M. An extension of Banach.s contraction principle Proc. Amer. Math. Soc., 7-10, 12 (1961).

[8] Edelstein M. On fixed and periodic points under contractive mappings J.London Math. Soc., 74-79, 37 (1962).

[9] Fisher B. Fixed point and constant mappings on metric spaces, Atti Accad, Naz., Lincci Rend. Ci. Sci. Mat. Natur. 61, 329-332 (1976).

[10] Fisher B., Khan M.S., Pairwise contractive mappings on Hausdorff space Bull. math. dela. Soc. Sci. math. delta R.S.de Roumanie Tome, 25(73) no.1, 37-40 (1981).

[11] Fisher B. On three fixed point mappings for compact metric spaces Indian J. Pure and Appl. Math. 8: 479-481 (1977).

[12] Gupta et al Some fixed point theorems for symmetric Hausdorff functions on Hausdorff spaces Appl. Math. Inf. Sci., 9(2), 833-839 (2015).

[13] Jaggi D.S., On common fixed points of contractive mapps Bull.Math. de la Soc. Sct. Math. de la R.S.R., 20, 143-146 (1976).

[14] Jinbiao H. Some fixed point theorems of Compact Hausdorff spaces Fixed point theory and Applications, 6, 67-69 (2007).

[15] Harinath H.K., A chain of results on fixed points Indian J. pure appl. Math., 1484-1490, 10(1979).

[16] Jungck G. Commuting mappings and fixed points Am. Math. Monthly. 83, 261-63 (1976).

[17] Mukerjee R.N. Common fixed points of some non linear mappings Indian J.purappl. Math. 12(8), 930-933 (1981).

[18] Pachpatte B.G. On certain Fixed point mapping in metric space, Journal of M.A.C.T Vol.13, 59-63 (1980).

[19] Pathak H.K. Some theorems on fixed points in pseudo compact tichonov space Indian J.pure appl. Math., 17(2), 180-1869 (1986).

[20] Rhoades R.E. Some theorems on weakly contractive maps, Nonlinear Anal., 47, 2483-2693. (2001).

[21] Sharma P.L. and Sahu M.K A unique fixed point theorem in complete metric space Acta Ciencia Indica Vol. XVII, M, 4, 685. (1991).

[22] Singh K.L. Contraction mappings and fixed point theorems Ann. Soc. Sci. Bruxelles, 83, 33-44. (1968).

[23] Yeh C.C., Common fixed point of continuous mappings in metric spaces Publ.Inst.Math.(Beograd) 27(41), 21-25. (1981). 